\title{
Adiposidade em adolescentes e obesidade materna
}

\author{
Relationship between maternal obesity \\ and adiposity in adolescents
}

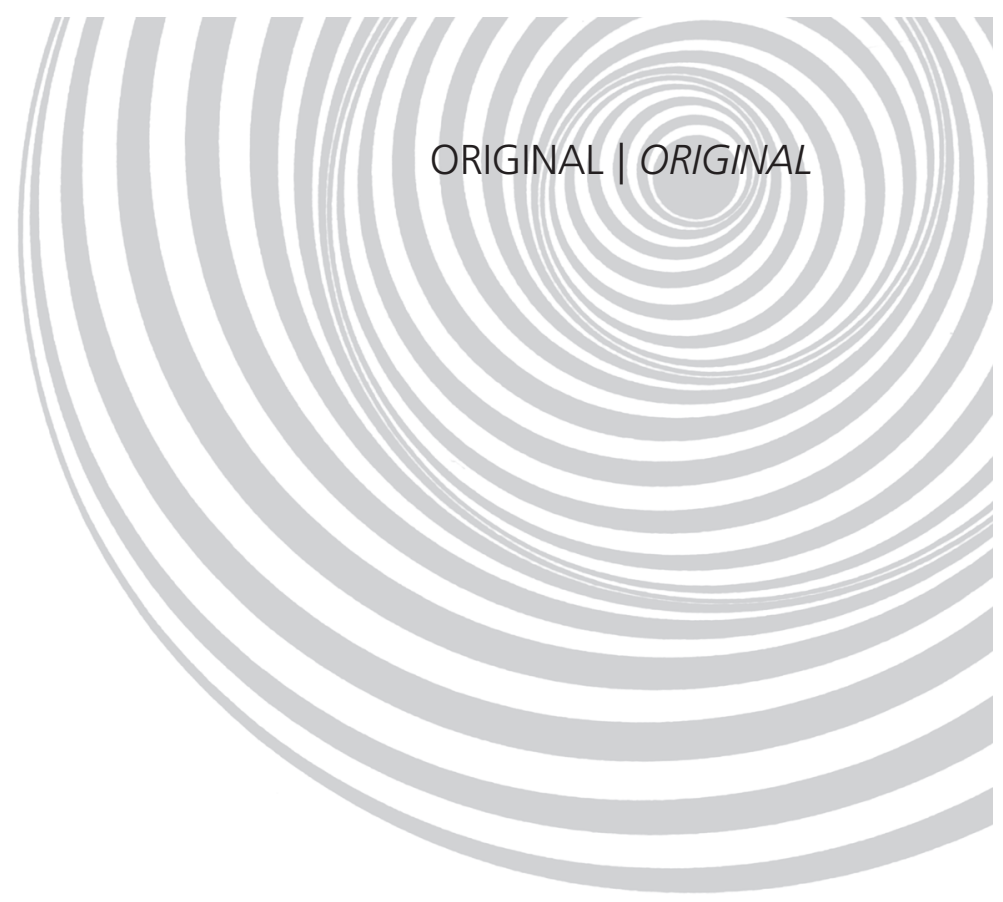

Maria Fernanda Petroli FRUTUOSO ${ }^{1}$

Ticiane Gonçalez BOVI²

Ana Maria Dianezi GAMBARDELLA ${ }^{3}$

RE S U M O

\section{Objetivo}

Descrever a relação entre adiposidade na adolescência e obesidade materna.

\section{Métodos}

Foi realizado estudo transversal com 660 indivíduos de 8 a 18 anos, de ambos os sexos, matriculados em uma escola pública e outra privada do município de São Paulo. A coleta de dados foi realizada por meio de entrevista, medidas antropométricas e inquérito alimentar. A adiposidade na adolescência foi mensurada a partir do índice de massa corporal e, por meio de análise de regressão, verificou-se sua relação com a obesidade materna, ajustada por sexo, idade, estágio de maturação sexual, valor energético total da dieta, atividade física, sedentarismo, peso ao nascer e escolaridade materna.

\section{Resultados}

Dos adolescentes estudados, 64,7\% eram do sexo feminino. A média (desvio-padrão) de idade foi de 12,4 $(1,80)$, variando de 8 a 17 anos. Verificou-se maior prevalência de excesso de peso e obesidade entre os indivíduos do sexo masculino, não sendo observada associação significativa entre estado nutricional e sexo. Após ajuste pelas covariáveis, detectou-se que filhos de mães obesas têm risco quatro vezes maior de ser obesos, quando comparados aos adolescentes filhos de mães não obesas.

\section{Conclusão}

Conclui-se que a obesidade materna representa fator de risco importante para o desenvolvimento da obesidade na adolescência.

Termos de indexação: Adolescência. Fatores de risco. Mães. Obesidade.

\footnotetext{
1 Universidade Federal de São Paulo, Curso de Nutrição, Departamento de Ciências da Saúde. Campus Baixada Santista, Av. Alm. Saldanha da Gama, 89, Ponta da Praia, 11030-400, Santos, SP, Brasil. Correspondência para/Correspondence to: M.F.P. FRUTUOSO. E-mail:<fernanda.frutuoso@unifesp.br>.

2 Universidade São Francisco, Curso de Nutrição. Bragança Paulista, SP, Brasil.

${ }^{3}$ Universidade de São Paulo, Faculdade de Saúde Pública, Departamento de Nutrição. São Paulo, SP, Brasil.
} 
6 | M.F.P. FRUTUOSO et al.

\section{A B S T R A C T}

\section{Objective}

This study aimed to describe the relationship between teenager's adiposity and maternal obesity.

\section{Methods}

A cross-sectional study was done with 660 teenagers aged 8 to 18 years, of both genders, students of private and public schools of São Paulo. The data were collected by interviews, anthropometric measurements and food intake records. Teenagers' adiposity was determined by body mass index and regression analyses was used to verify its relationship with maternal obesity adjusted for gender, age, stage of sexual development, energy intake, physical activity, sedentary lifestyle, birth weight and mother's education level.

\section{Results}

Most (64.7\%) of the teenagers were female. The mean age was 12.4 years (SD=1.80), aged 8 to 17 years. The prevalence of obesity and overweight was higher in boys. No statistical difference was found between nutritional status and gender. After the adjustments, the data show that children of obese mothers were 4 times more likely to be at risk of obesity than children of normal weight mothers.

\section{Conclusion}

Maternal obesity is a great risk factor for adolescent obesity.

Indexing terms: Adolescent. Risk factors. Mothers. Obesity.

\section{N T R O D U Ç Ã O}

Em decorrência de transformações biopsicossociais, a adolescência tornou-se um difícil, porém fascinante, período, que suscita grandes dúvidas e descobertas entre a comunidade científica, sobretudo pelas características do estado nutricional nessa fase, fortemente associado à qualidade de vida na idade adulta. Nesse sentido, estudos comprovam que o desenvolvimento de Doenças Crônicas Não-Transmissíveis (DCNT) e a obesidade na vida adulta têm raízes na infância ${ }^{1,2}$.

A obesidade em crianças e adolescentes está associada ao precoce aparecimento de moléstias crônicas não-transmissíveis, como diabetes Mellitus tipo 2, doenças cardiovasculares, distúrbios respiratórios, hipertensão arterial, dislipidemias, comprometimento postural e problemas psicológicos ${ }^{2,3}$.

Dados da Pesquisa de Orçamentos Familiares (POF), de 2002/2003, mostraram que a prevalência de excesso de peso em adolescentes brasileiros é crescente, atingindo o patamar de $16,7 \%$. No Brasil, estima-se que a prevalência do excesso de peso em adolescentes do sexo feminino tenha aumentado de 7,5\% em 1974-1975 para 15,4\% em 2002-2003, enquanto no sexo masculino o aumento foi de 3,9\% para $17,9 \%$ no mesmo período 4 .

Sob a influência familiar, social e publicitária, o adolescente tornou-se vulnerável a condutas errôneas, que podem influenciar não somente o seu hábito alimentar, mas principalmente o seu estado nutricional ${ }^{5}$. Além da influência familiar, outros fatores de risco podem colaborar para o aparecimento da obesidade na adolescência, sendo a inatividade física e o consumo alimentar inadequado duas variáveis comportamentais de grande importância para o aparecimento de excesso de peso. Segundo a Organização Mundial de Saúde ${ }^{6}$, a obesidade é um distúrbio nutricional que atinge todos os estratos socioeconômicos, porém tendente a apresentar maior frequência em famílias de baixo poder aquisitivo.

Estudos apontam que a maior ingestão de alimentos com elevado valor energético na adolescência coincide com o pico da velocidade máxima de crescimento e o aumento das necessidades energéticas. Entretanto, dietas com elevado valor energético podem desencadear efeitos deletérios à saúde do adolescente, contribuindo para o aumento da prevalência do excesso de peso ${ }^{7}$. 
O comportamento sedentário também pode contribuir para o acúmulo de gordura corporal. Estudos demonstram que, nas últimas 4 décadas, o nível de atividade física diminuiu entre os adolescentes, embora a ingestão dietética tenha permanecido inalterada 8 .

A maturação sexual constitui melhor variável, quando comparada à idade cronológica, para estratificação e análise do estado e conduta nutricional na adolescência, considerando que a puberdade promove uma série de transformações morfológicas e antropométricas, dentre as quais aumento da estatura, desenvolvimento muscular e até mesmo obesidade, influenciando diretamente a avaliação nutricional nesse grupo ${ }^{9}$.

Dentre os vários fatores de risco existentes, a obesidade dos pais, principalmente a materna, constitui um dos principais fatores de risco para o desenvolvimento da obesidade nos filhos. Filhos de pais obesos têm $80 \%$ de chance de também se tornarem obesos, enquanto tal proporção diminui para $40 \%$ quando apenas um dos genitores apresenta excesso de peso $1,2,6,8,9$.

Nesse contexto, este trabalho visa a descrever a relação entre adiposidade na adolescência e obesidade materna.

\section{M É T O D O S}

Para realização deste estudo transversal, foram coletados dados de 660 indivíduos de 8 a 18 anos, de ambos os sexos, matriculados em uma escola pública e outra privada do município de São Paulo. Com o intuito de obter uma população que contemplasse todos os estágios de maturação sexual (pré-púbere, púbere e pós-púbere) e considerando que no sexo feminino esta ocorre mais cedo que no masculino, foram incluídas meninas de 8 e 9 anos de idade na população estudada.

O cálculo do tamanho amostral foi realizado considerando-se um nível de significância de $95 \%$ e um poder da amostra (1- $\beta$ ) de $80 \%$. Baseando-se em estudos anteriores, estimou-se uma prevalência de adolescentes obesos de $8 \%$, com um valor de Odds Ratio (OR) de 2,5 para a variável obesidade materna (exposição) em relação ao desfecho (adiposidade dos adolescentes) e $30 \%$ de exposição entre os não obesos. Assim, estimou-se uma amostra de 585 indivíduos, acrescida de aproximadamente $20 \%$ para compensar possíveis perdas, totalizando 645 entrevistas. Os cálculos de tamanho amostral foram realizados com auxílio do programa Stata $10.0^{10}$. A seleção da amostra foi feita por meio de sorteio, em etapa única, após a ordenação do universo de alunos por série, sexo e idade.

A coleta de dados foi realizada pela pesquisadora, por meio de entrevistas e mensuração antropométrica, nas próprias instituições, entre agosto e outubro de 2007, sendo os dados registrados em formulários pré-testados.

Os dados referentes ao nível socioeconômico, peso e estatura das mães e peso dos filhos ao nascer foram obtidos por meio de questionário enviado aos pais, juntamente com o Termo de Consentimento. O projeto de pesquisa está de acordo com as normas da Resolução 196 do Conselho Nacional de Saúde, de 10/10/1996, que regulamenta as pesquisas envolvendo seres humanos, tendo sido aprovado pelo Comitê de Ética em Pesquisa da Faculdade de Saúde Pública da Universidade de São Paulo, com protocolo $n^{\circ} 427$, em 21/03/2001. Todos os participantes assinaram o Termo de Consentimento Livre e Esclarecido, concordando com a participação neste estudo.

Para a mensuração do peso corporal foi utilizada balança eletrônica do tipo plataforma (Tanita ${ }^{\circledR}$, com capacidade para $150 \mathrm{~kg}$ e graduação em 100g, e para a da estatura foi utilizado antropômetro $\left(\mathrm{Seca}^{\circledR}\right)$, com escala em milímetros, fixado em suporte de madeira, seguindo metodologia proposta por Gordon et al..11. Foi calculado o Índice de Massa Corporal (IMC) (IMC=peso/altura²).

A presença de obesidade foi verificada, entre as crianças e adolescentes estudados, segundo pontos de corte de IMC/idade e sexo, propostos por Cole et al. ${ }^{12}$. A obesidade foi classi- 
ficada como variável dependente do tipo categórica ( 0 = ausência; 1 = presença).

O índice de massa corporal das mães foi calculado a partir de peso e estatura referidos. Para a classificação de excesso de peso e obesidade utilizaram-se os pontos de corte recomendados pela Organização Mundial de Saúde, com IMC $\geq 25$ para sobrepeso e IMC $\geq 30$ para obesidade $^{6}$. O excesso de peso e a obesidade foram analisados como variáveis independentes categóricas ( 0 = ausência; $1=$ presença).

As covariáveis independentes utilizadas neste estudo foram nível socioeconômico (escolaridade materna), peso ao nascer e idade das crianças e adolescentes. Avaliou-se, ainda, estágio de maturação sexual, sedentarismo e consumo alimentar.

Para determinação do Estágio de Maturação Sexual (EMS) foi realizada autoavaliação, baseada na identificação por comparação com fotos de 5 etapas que caracterizam o desenvolvimento sexual da criança e adolescente, conforme critério proposto por Tanner, citado por Colli et al. ${ }^{13}$ : desenvolvimento das mamas para meninas, desenvolvimento dos genitais para meninos e de pelos pubianos para ambos os sexos.

Os indivíduos foram distribuídos em grupos pequenos do mesmo sexo e depois receberam informações sobre adolescência, desenvolvimento físico e maturação sexual, assim como explicações sobre a autoavaliação e o preenchimento do formulário. Uma vez que os indivíduos poderiam estar em fases diferentes para cada uma das características, visto que a maturação das mesmas obedece a mecanismos hormonais e genéticos diferentes ${ }^{13}$, adotou-se como critério de desenvolvimento o menor estágio de maturação sexual entre pelos pubianos e genitais para os meninos, e mamas e pelos pubianos para as meninas.

Investigou-se a prática de atividades físicas de lazer passivas, entendidas como a frequência e duração de atividades como assistir televisão, jogar videogame e utilizar o computador. Criou-se um escore de sedentarismo, composto pela soma do número de horas diárias assistindo tele- visão, jogando videogame e utilizando o computador.

Para a avaliação do consumo alimentar, foram realizados dois Recordatórios de 24 horas (R-24h), com o objetivo de obter informações sobre a quantidade de lipídeos ingeridos e o Valor Energético Total (VET) da dieta consumida pelas crianças e adolescentes. Os inquéritos alimentares foram realizados em dias não consecutivos. Para melhor descrição da porção consumida, foram utilizados álbum fotográfico ${ }^{14} \mathrm{e}$ utensílios domésticos.

Para o cálculo do valor energético total da dieta e da quantidade de lipídeos consumida, criou-se planilha com informações nutricionais do Estudo Nacional de Despesa Familiar (ENDEF) ${ }^{15}$. Foi incluída a composição nutricional de alimentos industrializados fornecida pelos fabricantes. O consumo de lipídeos foi ajustado pelo VET da dieta, conforme método proposto por Willet et al. ${ }^{16}$. Foram usados os resíduos da regressão, em que a variável dependente foi a quantidade de lipídeos consumida, e a independente, o VET da dieta.

Para a descrição das variáveis, foram utilizadas frequências, medidas de tendência central e dispersão. Utilizaram-se testes $t$ de Student e de Kruskal-Wallis para comparação entre as médias dos grupos. Os testes de associações entre as variáveis foram obtidos segundo a distribuição qui-quadrado e por meio do teste exato de Fischer. Estabeleceu-se nível de significância de 5\% para os testes estatísticos.

Para verificar a relação entre a adiposidade na adolescência e a obesidade materna, realizou-se análise de regressão logística, ajustada pelas covariáveis. Foram apresentados os valores de odds ratios e seus respectivos intervalos de confiança. Os cálculos estatísticos foram realizados com o auxílio do programa Stata $10.0^{10}$.

\section{RESULTADOS}

Os 660 indivíduos estudados apresentaram o Termo de Consentimento Livre e Esclarecido e 
a resposta ao Questionário, estando alguns destes incompletos devido ao fato de as crianças e adolescentes não residirem com pai e/ou mãe. Dessa forma, excluiu-se $23,9 \%$ da amostra inicial, dada a perda relacionada à ausência de informações solicitadas, perfazendo um total de 502 crianças e adolescentes.

Dos adolescentes estudados $64,7 \%$ eram do sexo feminino. A média (desvio-padrão) de idade foi de 12,4 (1,80), variando de 8 a 17 anos.

Segundo a Tabela 1, comparando-se os dois sexos, observam-se valores próximos de IMC, e superiores em peso e estatura para os meninos, sem diferença estatisticamente significante entre os sexos. Nota-se, ainda, maior prevalência de excesso de peso e obesidade entre os garotos, não sendo, porém, observada associação significativa entre estado nutricional e sexo.

Nota-se que a maioria das meninas encontra-se nos estágios finais de desenvolvimento puberal, ao contrário do observado entre os meninos. Foi detectada diferença estatisticamente significante para peso ao nascer $(p<0,05)$, mais elevado no sexo masculino (Tabela 2 ). As variáveis maternas apontam elevada escolaridade, prevalência de sobrepeso $(38,4 \%)$ e obesidade $(7,8 \%)$.

Tabela 1. Distribuição das variáveis antropométricas e de estado nutricional dos adolescentes segundo sexo. São Paulo (SP), 2008.

\begin{tabular}{|c|c|c|c|c|}
\hline \multirow{3}{*}{ Variável } & \multicolumn{4}{|c|}{$\operatorname{Sexo}(n=502)$} \\
\hline & \multicolumn{2}{|c|}{ Feminino $(n=35)$} & \multicolumn{2}{|c|}{ Masculino $(n=177)$} \\
\hline & $M$ & $\mathrm{DP}$ & $M$ & $\mathrm{DP}$ \\
\hline \multicolumn{5}{|l|}{ Antropométricas } \\
\hline Peso (kg) & 48,60 & 11,74 & 49,80 & 15,15 \\
\hline Estatura (m) & 153,40 & 8,25 & 154,80 & 12,22 \\
\hline IMC $\left(\mathrm{kg} / \mathrm{m}^{2}\right)$ & 20,40 & 3,75 & 20,30 & 4,23 \\
\hline Estado nutricional & \multicolumn{2}{|c|}{$\%$} & \multicolumn{2}{|c|}{$\%$} \\
\hline Excesso de peso & \multicolumn{2}{|c|}{26,10} & \multicolumn{2}{|c|}{30,20} \\
\hline Obesidade & \multicolumn{2}{|c|}{7,40} & \multicolumn{2}{|c|}{12,80} \\
\hline
\end{tabular}

M: média; DP: desvio-padrão.

Tabela 2. Distribuição das variáveis independentes segundo sexo. São Paulo (SP), 2008.

\begin{tabular}{|c|c|c|c|c|}
\hline \multirow{3}{*}{ Variável } & \multicolumn{4}{|c|}{$\operatorname{Sexo}(n=502)$} \\
\hline & \multicolumn{2}{|c|}{ Feminino $(n=325)$} & \multicolumn{2}{|c|}{ Masculino $(n=177)$} \\
\hline & $\mathrm{M}$ & DP & $\mathrm{M}$ & DP \\
\hline \multicolumn{5}{|l|}{ Demográficas e antropométricas } \\
\hline Idade (meses) & 154,60 & 21,34 & 153,40 & 19,53 \\
\hline Peso ao nascer ${ }^{*}$ & 3187,00 & 510,00 & 3283,00 & 590,70 \\
\hline Estágio de maturação sexual & \multicolumn{2}{|c|}{$\%$} & \multicolumn{2}{|c|}{$\%$} \\
\hline I- - || & \multicolumn{2}{|c|}{23,80} & \multicolumn{2}{|c|}{46,20} \\
\hline III & \multicolumn{2}{|c|}{41,40} & \multicolumn{2}{|c|}{25,30} \\
\hline $\mathrm{IV}-\mathrm{V}$ & \multicolumn{2}{|c|}{34,80} & \multicolumn{2}{|c|}{28,50} \\
\hline Estilo de vida & M & DP & M & DP \\
\hline Sedentarismo (h/dia) & 4,60 & 2,32 & 4,80 & 2,62 \\
\hline Valor Energético Total da dieta (kcal) & 1620,30 & 405,76 & 1685,90 & 350,99 \\
\hline Lipídeos da dieta (g) & 59,20 & 20,14 & 62,50 & 16,96 \\
\hline Variáveis maternas & $\mathrm{M}$ & DP & M & DP \\
\hline Escolaridade (anos) & 9,10 & 2,90 & 9,50 & 2,57 \\
\hline \multirow[t]{2}{*}{$\mathrm{IMC}\left(\mathrm{kg} / \mathrm{m}^{2}\right)$} & 24,60 & 3,47 & 24,80 & 3,70 \\
\hline & \multicolumn{2}{|c|}{$\%$} & \multicolumn{2}{|c|}{$\%$} \\
\hline Sobrepeso & \multicolumn{2}{|c|}{38,40} & \multicolumn{2}{|c|}{40,30} \\
\hline Obesidade & \multicolumn{2}{|c|}{7,80} & \multicolumn{2}{|c|}{8,00} \\
\hline
\end{tabular}

${ }^{*} p \leq 0,05$.

M: média; DP: desvio-padrão. 
Análise univariada mostrou associação somente entre obesidade na adolescência e obesidade materna $(p<0,000)$. As covariáveis foram então utilizadas no ajuste das análises, evidenciando que o risco de um adolescente ser obeso quando a mãe é 5,34 vezes o risco de obesidade em um adolescente filho de mãe não obesa (Tabela 3).

Modelos de regressão utilizando idade cronológica em lugar do estágio de maturação sexual não apresentaram diferenças importantes em relação ao modelo apresentado na Tabela 3.

\section{S C U S S Ã O}

A obesidade é resultado do acúmulo excessivo de tecido adiposo, proveniente de dietas com elevado valor energético total e baixo dispêndio energético diário ${ }^{6}$. Vários fatores podem influenciar o desenvolvimento da obesidade na adolescência, a saber, consumo alimentar inadequado, inatividade física, estágio de maturação sexual, nível socioeconômico e influência parental.

A adolescência é um período caracterizado pela mudança no padrão alimentar, no qual há um elevado consumo de alimentos com alto teor de gordura, carboidratos refinados e sódio, assim como baixa ingestão de frutas, verduras e legumes, além da adoção de modismos alimentares, dietas monótonas e omissão de refeições. Tais condutas estão sendo associadas a diversos distúrbios nutricionais, dentre os quais a obesida$\mathrm{de}^{2,7,8,17}$.

Tabela 3. Razões de chances para variáveis estudadas segundo análise de regressão bruta e ajustada para adiposidade na adolescência. São Paulo (SP), 2008.

\begin{tabular}{|c|c|c|}
\hline \multirow{2}{*}{ Variável } & \multicolumn{2}{|c|}{ Odds ratio [intervalo de confiança] } \\
\hline & Bruto & Ajustado \\
\hline \multicolumn{3}{|l|}{ Sexo } \\
\hline Masculino & 1,00 & 1,00 \\
\hline Feminino & $0,56[0,32-0,91]$ & $0,61[0,31-1,21]$ \\
\hline \multicolumn{3}{|c|}{ Peso ao nascer } \\
\hline$\leq 2500 \mathrm{~g}$ & $1,05[0,39-2,78]$ & $1,06[0,38-2,94]$ \\
\hline$>2500 \mathrm{~g}$ & 1,00 & 1,00 \\
\hline \multicolumn{3}{|c|}{ Estágio de maturação sexual } \\
\hline Pré-púbere & $1,05[0,98-1,09]$ & $1,02[0,92-1,36]$ \\
\hline Púbere & $1,69[0,75-3,80]$ & $1,65[0,70-3,89]$ \\
\hline Pós-púbere & 1,00 & 1,00 \\
\hline \multicolumn{3}{|c|}{ Sedentarismo } \\
\hline$\leq 4 \mathrm{~h} / \mathrm{dia}$ & 1,00 & 1,00 \\
\hline$>4 \mathrm{~h} / \mathrm{dia}$ & $1,11[0,58-2,11]$ & $1,11[0,57-2,14]$ \\
\hline \multicolumn{3}{|c|}{ Valor energético total da dieta (kcal) } \\
\hline$\leq 1650$ & 1,00 & 1,00 \\
\hline$>1650$ & $1,39[0,73-2,64]$ & $1,93[0,82-4,55]$ \\
\hline \multicolumn{3}{|l|}{ Lipídeos (g) } \\
\hline$\leq 60$ & 1,00 & 1,00 \\
\hline$>60$ & $0,92[0,48-1,73]$ & $0,58[0,24-1,34]$ \\
\hline \multicolumn{3}{|c|}{ Escolaridade (anos) } \\
\hline$\leq 5$ & $0,75[0,28-1,97]$ & $0,71[0,25-2,01]$ \\
\hline$>5$ & 1,00 & 1,00 \\
\hline \multicolumn{3}{|c|}{ Obesidade materna } \\
\hline $\operatorname{Sim}$ & $4,84[2,15-10,89]$ & $5,34[2,30-12,38]$ \\
\hline Não & 1,00 & 1,00 \\
\hline
\end{tabular}


No que diz respeito ao consumo alimentar inadequado e à inatividade física, as maiores dificuldades e limitações em estudos transversais sobre alimentação/atividade física/adiposidade consistem em mensurar o efeito cumulativo do excesso de valor energético e o baixo gasto energético na composição corporal.

Ao avaliarem a dieta habitual de crianças e adolescentes com sobrepeso e obesidade, Lima et al. ${ }^{18}$ identificaram diferença significativa no consumo de proteínas e lipídeos entre adolescentes com excesso de peso e o grupo-controle, com maior ingestão desses macronutrientes no primeiro grupo.

No presente estudo, o consumo médio de lipídeos correspondeu a aproximadamente 32\% do valor energético total da dieta entre as meninas e 33,3\% entre os meninos, sendo que tal valor não deveria ultrapassar $30,0 \%$, segundo proposto pela Dietary Reference Intakes ${ }^{19}$.

Os adolescentes informaram permanecer por mais de 4 horas em atividades sedentárias, como assistir televisão, jogar videogame e utilizar o computador, condutas que podem ter provocado maior prevalência de sobrepeso e obesidade, principalmente no sexo masculino, que apresentou maior consumo de lipídeos e maior período em atividades sedentárias. Estudo realizado por Frutuoso et al..$^{20}$ mostrou associação entre assistir TV por mais de duas horas diárias e apresentar sobrepeso, especialmente entre meninos.

Campagnolo et al. ${ }^{21}$ relatam que adolescentes com o hábito de assistir TV por mais de 5 horas/dia têm probabilidade três vezes maior de desenvolver obesidade quando comparados a adolescentes que assistem TV por menos de 2 horas/dia. Esses adolescentes estariam ainda sujeitos à influência da propaganda de alimentos, principalmente aqueles com elevada densidade energética, além de desenvolver o hábito de comer em frente à televisão, fato que contribuiria para o aumento da obesidade nessa faixa etária.

A maturação sexual representa outro fator de grande valia para avaliação do crescimento e desenvolvimento do adolescente. A puberdade promove uma série de modificações em relação à massa magra e na distribuição da gordura corporal, com impacto imediato no estado nutricional. Adolescentes que maturam sexualmente mais tarde apresentam peso mais baixo e estatura mais elevada, quando comparados àqueles que maturaram precocemente. Desse modo, o risco de desenvolvimento de obesidade é maior entre aqueles que atingem precocemente o maior nível de maturação sexual2,9,12,13,17.

Estudos com adolescentes de 7 a 17 anos de idade, de ambos os sexos, mostram que o IMC aumenta cerca de 0,5 a $1,3 \mathrm{~kg} / \mathrm{m}^{2}$ a cada estágio de maturação sexual ${ }^{22}$. No presente estudo, adolescentes do sexo feminino encontram-se nos estágios mais avançados da puberdade, enquanto os do sexo masculino encontram-se nas fases iniciais. Esse dado pode implicar maior quantidade de gordura corporal no sexo feminino e consequentemente maior IMC, pois o aumento da massa de gordura, que ocorre durante a puberdade em ambos os sexos, é mais lento no masculino e superado pelo ganho de massa livre de gordura.

A literatura não apresenta consenso no que diz respeito à confiabilidade da autoavaliação na determinação do EMS, que é verificada, na totalidade dos estudos, pelo coeficiente de concordância entre o estágio da autoavaliação e o estágio apontado por profissional especializado.

Duke et al. ${ }^{23}$, avaliando adolescentes norte-americanos de ambos os sexos, encontraram para as meninas coeficiente kappa de 0,81 para o desenvolvimento das mamas e 0,91 para pelos pubianos e, para os meninos, coeficiente de 0,88 para um escore combinando o desenvolvimento dos genitais e pelos pubianos.

No Brasil, Guimarães \& Passos ${ }^{24}$ analisaram a concordância entre informações referidas e observadas do EMS de meninas. Os percentuais de concordância para desenvolvimento das mamas variaram de 57,3 a $65,2 \%$ e, para os pelos pubianos, entre 65,4 e 73,5\%, valores inferiores aos apresentados na literatura, segundo os autores. Matsudo e Matsudo, citados por Sun et al. ${ }^{25}$, 
apresentam concordância entre autoavaliações repetidas, variando entre 85 e 95\%, em estudos com adolescentes brasileiros.

No presente estudo, descrições detalhadas sobre as diferenças entre os estágios de maturação sexual foram utilizadas com o intuito de facilitar a autoavaliação e minimizar erros.

Algumas variáveis relacionadas aos pais e/ou família, como estado nutricional e nível socioeconômico podem influenciar o desenvolvimento da obesidade na infância e na adolescência. Terres et al. ${ }^{2}$ relataram que o maior fator de risco de obesidade nas crianças é a presença da mesma em seus pais, graças à soma dos fatores genéticos e ambientais, apontando a existência de uma "transmissão familiar da obesidade".

Mendes et al. ${ }^{1}$ apontaram os fatores genéticos como justificativa para os achados de maior prevalência de sobrepeso em filhos de pais com sobrepeso, sem no entanto descartar que fatores ambientais, como hábitos alimentares inadequados e inatividade física dos pais, possam ser incorporados como exemplo pelos filhos.

O risco aumentado de obesidade nos adolescentes estudados, na presença de obesidade materna, evidencia a influência do estado nutricional materno na prole. Estudos com gêmeos segregados e indivíduos adotados mostram que o risco de obesidade é maior entre descendentes de obesos ${ }^{6}$.

Frutuoso $^{17}$ e Engstrom \& Anjos $^{26}$ identificam o excesso de peso ou a obesidade materna como fator de grande influência sobre o estado nutricional de crianças e adolescentes. Segundo os primeiros ${ }^{26}$, mães e filhos compartilham das mesmas condições socioambientais, culturais e de hábitos alimentares e, consequentemente, tais fatores podem influenciar o estado nutricional de ambos, sendo 3,19 vezes maior o risco de uma criança apresentar sobrepeso quando a mãe é obesa. Fernandes et al. ${ }^{27}$ evidenciaram que a obesidade da mãe aumenta o risco dos filhos adolescentes apresentarem acúmulo de gordura abdominal.
Também a escolaridade materna é abordada como um dos principais fatores de influência sobre o estado nutricional de crianças e adolescentes. Neste estudo, este item foi relativamente alto (cerca de 9 anos), confirmando os achados que apontam relação entre a escolaridade materna e o estado nutricional do adolescente. Estudos nacionais apontam que crianças e adolescentes com mães de maior escolaridade passam mais tempo em frente à TV, o que contribuiria para o aumento do sedentarismo e, consequentemente, da obesidade ${ }^{21,28}$. Tal situação, muito embora não pareça condizente com o nível de informação das mães, pode ser explicada pela tendência das mesmas em restringir as atividades dos filhos, por medo da violência urbana.

Além disso, mães com maior nível de escolaridade tendem a se inserir no mercado de trabalho, melhorando as condições socioeconômicas da família, o que pode provocar mudança no padrão alimentar e na rotina familiar, priorizando-se a facilidade em detrimento da saúde dos adolescentes. Salvador ${ }^{28}$ verificou que, apesar de mães com maior nível socioeconômico apresentarem maior probabilidade de acesso a informações sobre a relação entre alimentação/atividade física/obesidade, esse conhecimento não se reflete no estado nutricional dos filhos.

Em estudo com pré-escolares, escolares e adolescentes de diferentes condições socioeconômicas, Silva et al. ${ }^{3}$ concluíram que indivíduos com melhor poder aquisitivo estão mais suscetíveis ao excesso de peso e à obesidade. Adolescentes com maior nível socioeconômico possuem maior acesso às informações e a atividade física estruturada, mas ao mesmo tempo estão mais suscetíveis ao consumismo e aos novos aparatos tecnológicos que contribuem para o sedentarismo.

Já para os adolescentes de menor nível socioeconômico, o acesso à informação seria mais restrito, principalmente pela menor escolaridade, o que limitaria os conhecimentos sobre saúde. A menor adesão desse grupo à prática de atividade física estaria ligada à inserção do adolescente no mercado de trabalho, o que propiciaria a reali- 
zação de atividades ocupacionais de grande dispêndio energético. Adicionalmente, esses adolescentes estariam expostos a um novo padrão alimentar, com predomínio de produtos com elevada densidade energética e menor custo, e ao reduzido consumo de frutas, verduras e legumes ${ }^{29}$.

Estudos recentes demonstram que inúmeras doenças, como diabetes Mellitus tipo 2, coronariopatias, acidente vascular cerebral, hipertensão arterial e principalmente a obesidade, possuem forte associação com o baixo peso dos indivíduos ao nascer ${ }^{30}$. A desnutrição intrauterina provoca uma série de mecanismos adaptativos, tanto endócrinos quanto metabólicos, que buscam a sobrevivência do indivíduo. Entretanto, após o término desse período, com a regularização da oferta de nutrientes, aquela adaptação provocaria uma série de feitos adversos à saúde, dentre eles a obesidade e a intolerância à glicose $\mathrm{e}^{30,31}$.

Entretanto, observa-se uma dicotomia entre os resultados apresentados pelos estudos que abordam o tema, na medida em que algumas investigações apontam o elevado peso ao nascer, em especial valores superiores a 4 mil gramas, como fator de risco para o desenvolvimento da obesidade na vida adulta ${ }^{32,33}$.

Dados do presente estudo identificaram maior peso ao nascer no sexo masculino, o qual também apresentou maior prevalência tanto de excesso de peso quanto de obesidade. Dessa forma, mostrou-se positiva a associação entre peso elevado ao nascer (peso >2500g) e obesidade na adolescência.

Entretanto, uma das limitações deste estudo quanto à associação entre peso ao nascer e obesidade na adolescência pode ser apontada pela utilização daquele dado a partir da informação dos pais. Portanto, tal variável pode ter sofrido um viés de memória, que alteraria o resultado final apresentado pela amostra. Diante disso, estudos que visem à correção dessa variável antropométrica são de fundamental importância.

O presente estudo também utilizou, para classificação da obesidade materna, medidas antropométricas referidas pelas mães. A utilização de medidas autorrelatadas tem sido abordada com frequência em diversos estudos de avaliação do estado nutricional em adultos como um método prático, de baixo custo e adequado para monitorização da prevalência de obesidade na população. Entretanto, a literatura relata que medidas autorreferidas sofrem influência de vários fatores, como sexo, idade, renda familiar e escolaridade, devendo ser usadas com cautela ${ }^{34-36}$.

Peixoto et al. ${ }^{35}$ sugerem a realização de estudos para o reconhecimento da magnitude dos erros antes da utilização das medidas referidas, enquanto Silveira et al. ${ }^{36}$ acreditam que a correção do IMC referido minimizaria os erros, tornando os dados confiáveis.

No presente trabalho, a fim de corrigir a informação referida (ainda que não fosse esse o seu objetivo principal), foram realizadas medições de peso e estatura em uma sub-amostra de mães ( $n=167)$ que participaram de reuniões de pais e mestres. Não foram observadas diferenças significativas entre a medida aferida e a informada, sendo as diferenças médias de $-0,23 \mathrm{~kg} \mathrm{e}+0,47 \mathrm{~cm}$. Tal achado permitiu corrigir as medidas referidas e garantir, neste estudo, a confiabilidade das medidas maternas de peso e estatura, que não constituíram limitação para as análises.

Conclui-se que a obesidade materna representa fator de risco importante para o desenvolvimento da obesidade na adolescência. A possibilidade de influência materna, tanto na esfera genética quanto socioambiental, requer estudos sobre a magnitude de seus efeitos sobre a saúde da mãe e de sua prole, de modo a fornecer subsídios para programas e ações de prevenção primária da obesidade ao longo do ciclo de vida.

\section{COLABORADORES}

M.F.P. FRUTUOSO, T.G. BOVI e A.M.D.D. GAMBARDELLA contribuíram para a análise dos dados, discussão dos resultados e redação do artigo. 


\section{REFERÊ NCIAS}

1. Mendes MJFL, Alves JGB, Alves AV, Siqueira PP, Freire EFC. Associação de fatores de risco para doenças cardiovasculares em adolescentes e seus pais. Rev Bras Saúde Mater Infant. 2006; 6(Supl 1): S49-S54.

2. Terres NG, Pinheiro RT, Horta BL, Pinheiro KAT, Horta LL. Prevalência e fatores associados ao sobrepeso e à obesidade em adolescentes. Rev Saúde Pública. 2006; 40(4):627-33.

3. Silva GAP, Balaban G, Motta MEFA. Prevalência de sobrepeso e obesidade em crianças e adolescentes de diferentes condições socioeconômicas. Rev Bras Saúde Mater Infant. 2005; 5(1):53-9.

4. Instituto Brasileiro de Geografia e Estatística. Pesquisa de orçamentos familiares 2002-2003: antropometria e análise do estado nutricional de crianças e adolescentes no Brasil. Brasília: IBGE; 2006.

5. Boog MCF, Vieira CM, Oliveira NL, Fonseca O, L'Abbate S. Utilização de vídeo como estratégia de educação nutricional para adolescentes: comer... o fruto ou o produto? Rev Nutr. 2003; 16(3): 281-93. doi: 10.1590/S1415-52732003000300 006.

6. World Health Organization. Obesity: preventing and managing the global epidemic. Geneva: WHO; 2000. Technical Report Series, 894.

7. Enes CC, Slater B. Obesidade na adolescência e seus principais fatores determinantes. Rev Bras Epidemiol. 2010; 13(1):163-71.

8. Suñé FR, Dias-da-Costa JS, Olinto MTA, Pattussi MP. Prevalência e fatores associados para sobrepeso e obesidade em escolares de uma cidade no Sul do Brasil. Cad Saúde Pública. 2007; 23(6):1361-71.

9. World Health Organization. Physical status: the use and interpretation of anthropometry. Geneve: WHO; 1995. Technical Report Series, 854.

10. Statacorp. Stata Statistical Software: Release 10.0. College Station (TX): Stata Corporation; 2008.

11. Gordon CC, Chumlea WC, Roche AF. Stature, recumbent lenght, and weight. In: Lohman TG, Roche AF, Martorell R. Anthropometric standardization reference manual. Champaign: Human Kinetics Books; 1988. p.3-8.

12. Cole TJ, Bellizi MC, Flegal KM, Dietz WH. Establishing a standard definition for child overweight and obesity worldwide: international survey. BMJ. 2000; 320(5):1240-3.

13. Colli AS, Coates V, Guimarães EMB. Monitoração do crescimento e desenvolvimento físico. In: Coates V. Medicina do adolescente. São Paulo: Sarvier; 1993. p.51-65.
14. Zabotto CB, Vianna RPT, Gil MF. Registro fotográfico para inquéritos dietéticos: utensílios e porções. Goiânia: UFG; 1996.

15. Instituto Brasileiro de Geografia e Estatística. Estudo nacional de despesa familiar; tabela de composição de alimentos. Rio de Janeiro: IBGE; 1996.

16. Willet WC, Howe GR, Kushi LH. Adjustment for total energy intake in epidemiologic studies. Am J Clin Nutr. 1997; 65(supl):1220S-8S.

17. Frutuoso MFP. Fatores associados à obesidade em indivíduos de 8 a 18 anos de idade [doutorado]. São Paulo: Universidade de São Paulo; 2003.

18. Lima SCVC, Arrais RF, Pedrosa LFC. Avaliação da dieta habitual de crianças e adolescentes com sobrepeso e obesidade. Rev Nutr. 2004; 17(4):469-77. doi: 10.1590/S1415-52732004000400007.

19. Institute of Medicine. Dietary reference intake for energy, carbohydrates, fiber, fat, protein and acids (macronutrients). Washington (DC): National Academy Press; 2002.

20. Frutuoso MFP, Bismarck-Nasr EM, Gambardella AMD. Redução do dispêndio energético e excesso de peso corporal em adolescentes. Rev Nutr. 2003; 16(3):257-63. doi: 10.1590/\$1415-52732003000 300003.

21. Campagnolo PDB, Vitolo MR, Gama CM. Fatores associados ao hábito de assistir TV em excesso entre adolescentes. Rev Bras Med Esporte. 2008; 14(3): 197-200.

22. Barbosa KBF, Franceschini SCC, Priore SE. Influência dos estágios de maturação sexual no estado nutricional, antropometria e composição corporal de adolescentes. Rev Bras Saude Mater Infant. 2006; 6(4):375-82.

23. Duke PM, Litt IF, Gross RT. Adolescents' selfassessment of sexual maturation. Pediatrics. 1980; 66(6):918-20.

24. Guimarães JP, Passos ADC. Análise da concordância entre informações referidas e observadas acerca do estadiamento pubertário entre escolares do sexo feminino. Rev Saúde Pública. 1997; 31(3): 263-71.

25. Sun SS, Schubert CM, Chumlea WC, Roche AF, Kulin $\mathrm{HE}$, Lee PA, et al. National estimates of the timing of sexual maturation and racial differences among US children. Pediatrics. 2002; 110:911-9.

26. Engstrom EM, Anjos LA. Relação entre o estado nutricional materno e sobrepeso nas crianças brasileiras. Rev Saúde Pública. 1996; 30(3):233-9.

27. Fernandes RA, Casonatto J, Christofaro DGD, Cucato GG, Oliveira AR, Freitas Junior IF. Fatores familiares associados à obesidade abdominal entre adolescentes. Rev Bras Saúde Mater Infan. 2009; 9(4):451-7. 
28. Salvador CCZ. Fatores associados ao estado nutricional dos estudantes da $4^{a}$ à $9^{a}$ série do ensino fundamental de 3 regiões administrativas do município de Vitória [doutorado]. São Paulo: Universidade de São Paulo; 2008.

29. Campos LA, Leite AJM, Almeida PC. Nível socioeconômico e sua influência sobre a prevalência de sobrepeso e obesidade em escolares adolescentes do município de Fortaleza. Rev Nutr. 2006; 19(5): 531-8. doi: 10.1590/S1415-52732006000500001.

30. Silveira VMF, Horta BL. Peso ao nascer e síndrome metabólica em adultos: meta-análise. Rev Saúde Pública. 2008; 42(1):10-8.

31. Bismarck-Nasr EM, Frutuoso MFP, Gambardella AMD. Efeitos tardios do baixo peso ao nascer. Rev Bras Crescimento Desenvolv Hum. 2008; 18(1): 80-5.

32. Martins EB, Carvalho MS. Associação entre peso ao nascer e o excesso de peso na infância: revisão sistemática. Cad Saúde Pública. 2006; 22(11): 2281-300.
33. Bismarck-Nasr EM, Frutuoso MFP, Gambardella AMD. Relação entre índice ponderal ao nascer e excesso de peso corporal em jovens. Cad Saúde Pública. 2007; 23(9):2064-71.

34. Fonseca MJM, Faerstein E, Chor D, Lopes CS. Validade de peso e estatura informados e índice de massa corporal: estudo pró-saúde. Rev Saúde Pública. 2004; 38(3):392-8.

35. Peixoto MRG, Benício MHDA, Jardim PCBV. Validade do peso e da altura auto-referidos: o estudo de Goiânia. Rev Saúde Pública. 2006; 40(6): 1065-72.

36. Silveira EA, Araújo CL, Gigante DP, Barros AJD, Lima MS. Validação do peso e altura referidos para o diagnóstico do estado nutricional em uma população de adultos no Sul do Brasil. Cad Saúde Pública. 2005; 21(1):235-45.

Recebido em: 27/3/2009

Versão final reapresentada em: 21/7/2010

Aprovado em: 16/8/2010 
